\title{
Dysregulated connexin 43 in HER2-positive drug resistant breast cancer cells enhances proliferation and migration
}

\author{
Elizabeth S. Yeh ${ }^{1}$, Christina J. Williams ${ }^{1}$, Carly Bess Williams ${ }^{1}$, Ingrid V. Bonilla, \\ Nancy Klauber-DeMore ${ }^{2}$ and Stephanie L. Phillips ${ }^{3}$ \\ ${ }^{1}$ Department of Cell and Molecular Pharmacology and Experimental Therapeutics, Medical University of South Carolina, \\ Charleston, SC, USA \\ ${ }^{2}$ Department of Surgery, Medical University of South Carolina, Charleston, SC, USA \\ ${ }^{3}$ Department of Pediatrics, Division of Pediatric Hematology/Oncology, Medical University of South Carolina, Charleston, SC, \\ USA \\ Correspondence to: Elizabeth S. Yeh, email: yeh@musc.edu \\ Keywords: connexin 43; gap junctions; HER2+ breast cancer; cell migration; resistance \\ Received: May 17, $2017 \quad$ Accepted: November 01, $2017 \quad$ Published: November 25, 2017 \\ Copyright: Yeh et al. This is an open-access article distributed under the terms of the Creative Commons Attribution License 3.0 \\ (CC BY 3.0), which permits unrestricted use, distribution, and reproduction in any medium, provided the original author and source \\ are credited.
}

\section{ABSTRACT}

Connexin $43(\mathrm{Cx} 43)$ is a gap junction protein whose function in the development of breast cancer and in breast cancer progression remains unclear. Evidence suggests that Cx43 (GJA1) mRNA and protein expression is altered in breast tumors. However, reports indicate both increased and decreased Cx43 levels in human breast cancer samples. Studies also suggest that loss of Cx43 regulated gap junction intercellular communication is a common feature of breast malignancies that potentially correlates with histological stage. Further evidence suggests that Cx43 (GJA1) mRNA expression is negatively correlated with HER2 positivity but a relationship between Cx43 and HER2 in breast cancer is not well defined. Therefore, in this study, we sought to evaluate the relationship between Cx43 activity, HER2, and drug resistance. Using HER2 + breast cancer cell lines that are sensitive or resistant to HER2 inhibitor, we evaluated Cx43 gap junction function. We found that Cx43 gap junction activity is completely lost in drug resistant HER2-positive (HER2+) breast cancer cells, whereas Cx43 gap junction activity can be restored by $\mathrm{Cx} 43$ overexpression in drug sensitive HER2+ cells. Moreover, the dysregulation of Cx43 resulted in increased tumorigenic and migratory capacity of the HER2+ drug resistant breast cancer cells.

\section{INTRODUCTION}

Connexins are the primary protein components of gap junctions, which are structures composed of aggregates of intercellular channels that facilitate direct cell-to-cell communication. More than 50 years ago, Loewenstein and colleagues first published that cancer cells lose their ability to communicate via gap junctions, highlighting the importance of intercellular communication for normal cell homeostasis [1]. Consequently, a potential benefit of targeting connexins in cancer is the ability to restore cell-to-cell communication, and possibly gap junction-mediated propagation of death signals, the so-called bystander effect. Combining this property with a drug cocktail has the potential to amplify the desired chemotherapeutic properties of cancer agents as well as reversing the inherent cancer promoting cellular properties of improperly functioning connexins.

Connexin $43(\mathrm{Cx} 43)$ is arguably the most well studied of the connexin family members in breast cancer. Reports from studies examining human breast cancer tissue samples indicate that levels of $\mathrm{Cx} 43$ both increase and decrease with breast cancer stage [2-5]. In terms of activity, the current prevailing theory is that 
Cx43 gap junction intercellular communication (GJIC) is reduced in early stages of breast cancer as well as during the initial metastatic steps $[6,7]$. Experimental studies suggest that reduced $\mathrm{Cx} 43$ expression levels or a diminished capacity for $\mathrm{Cx} 43$-dependent GJIC promotes breast cancer cell migration. [8-15]. It has also been suggested that $\mathrm{Cx} 43$ gap junctional intercellular communication between tumor cells and vasculature is potentially facilitative during later stages of metastasis involving extravasation and colonization in the secondary metastatic site [16-19]. Other areas of experimental investigation highlight non-canonical (i.e. non-gap junction related) functions for $\mathrm{Cx} 43$ and their importance in regulating cellular functions including cancer cell migration [20].

A major roadblock in furthering $\mathrm{Cx} 43$ research in breast cancer, as well as other human cancers, arises from the standard of using expression levels either at the genomic or protein level as a surrogate for $\mathrm{Cx} 43$ activity. While potentially useful, the idea that expression levels directly corresponds to activity levels may be inaccurate. Increased Cx43 levels do not necessarily equate with increased GJIC, particularly if the increase in Cx43 is coupled with cytoplasmic localization of the protein. Our current studies highlight this problem and serve to illustrate that there is a more complicated system of regulation that should be investigated in detail to appropriately facilitate $\mathrm{Cx} 43$-specific drug targeting and translation.

Previous evidence suggests that Cx43 expression levels are negatively correlated with HER2 expression $[21,22]$. Additional experimental evidence indicates that HER2+ breast cancer cell lines have reduced Cx43 expression as compared to ER+ breast cancer cells $[23,24]$. Somewhat paradoxically, the analysis presented here, suggests that high Cx43 expression levels correlates with reduced relapse free survival (RFS) in HER2-positive (HER2+) breast cancer. Since neither the former nor the latter analyses assessed $\mathrm{Cx} 43$ activity, we initiated an investigation to evaluate $\mathrm{Cx} 43$ protein activity and GJIC in HER2+ breast cancer cells. We posited that in HER2+ breast cancer cells that are responsive to HER2 inhibitors, GJIC could be restored by modulation of $\mathrm{Cx} 43$. However, in drug resistant cells the right opportunities or conditions are less favorable because additional mechanisms have arisen in drug resistant cells that further block $\mathrm{Cx} 43 \mathrm{GJIC}$. To test this idea, we evaluated $\mathrm{Cx} 43$ function in HER2 inhibitor sensitive and resistant breast cancer cells and found that in fact, the drug resistant cells were clearly incompetent in their ability to propagate Cx43 GJIC. We put forth the conjecture that the more severe phenotype of $\mathrm{Cx} 43$ dysregulation in drug resistant cells is likely detrimental due to the cancer promoting capacity of losing GJIC, not necessarily directly from the loss of GJIC, but rather from the shift towards non-canonical functions for $\mathrm{Cx} 43$.

\section{RESULTS}

\section{Different levels of Cx43 (GJAI1) mRNA but not protein are expressed in drug sensitive and drug resistant HER2+ breast cancer cells}

To evaluate the significance of $\mathrm{Cx} 43$ gene expression levels in HER2+ breast cancer, we used the Kaplan-meier plotter database tool (kmplot.com) to assess whether Cx43 (GJA1) gene expression correlates with relapse free survival (RFS) in HER2+ patients [25]. Using this tool, a gene probe for GJA1 (201667_at) was used for analysis with HER2 status set to "positive" and ER status set to "negative" yielding $n=137$ patient samples with available clinical data containing the selected events. A total of $\mathrm{n}=68$ patients were scored as "low" GJA1 and $\mathrm{n}=69$ were scored as "high" GJA1. The analysis tool automatically removed redundant samples and excluded any biased arrays. The probe expression range was classified by the Km plotter tool as 73-16584 with a cutoff value of 2320 used for analysis. Our analysis showed that GJA1 expression correlates with reduced RFS (Figure 1A), which is somewhat inconsistent with previously published evidence suggesting that Cx43 expression levels are negatively correlated with HER2 expression $[21,22]$. Since the expression analysis does not evaluate Cx43 protein levels or function, we asked whether the regulation of these aspects could differ between a HER2+ cell line that was sensitive to HER2 inhibitors compared to one that was resistant. Therefore, we first evaluated Cx43 (GJA1) gene expression by quantitative RealTime PCR in SK-BR-3 cells (drug sensitive) and JIMT-1 cells (drug resistant), two commonly used HER2+ cell models. Consistent with the gene expression analysis where higher expression of $\mathrm{Cx} 43$ correlated with poorer outcome (Figure 1A), GJA1 levels in the drug resistant JIMT-1 cells were higher than the drug sensitive SK-BR-3 cells (Figure 1B). However, when we evaluated endogenous $\mathrm{Cx} 43$ protein expression and compared this between cell lines, there was no difference in $\mathrm{Cx} 43$ protein levels (Figure 1C and Supplementary Figure 1). These findings suggested to us that $\mathrm{Cx} 43$ has multiple nodes of regulation in breast cancer cells and evaluating gene expression is potentially not indicative of protein regulation or function.

\section{HER2 inhibitor resistant breast cancer cells are gap junction deficient}

To determine if either of the HER2+ cell lines are Cx43 GJIC competent, we first evaluated baseline gap junction activity in each cell line using a parachute cell coupling assay. For this assay, 5000 "donor" cells were loaded with a cell permeable dye called Calcein AM. These cells were then dropped onto "acceptor" cells to evaluate dye transfer from the donor cells to the acceptor cells. Interestingly, neither the SKBR3 (sensitive) nor the 
JIMT-1 (resistant) cell line had any baseline gap junction activity since neither had increased dye transfer above the initial 5000 cells that were loaded with Calcein AM as donor cells (Supplementary Figure 2). This observation is consistent with the idea the HER2+ breast cancer cells reduce their $\mathrm{Cx} 43$ protein expression levels resulting in impaired GJIC.

We next overexpressed $\mathrm{Cx} 43$ in each cell line by stable retroviral transduction because prior reports indicate that overexpression is a method that can drive the restoration of GJIC in breast cancer cell lines [19, 26-28]. We also stably transduced cells with a mutant of Cx43 that contains a Glycine to Serine amino acid substitution, G60S. This mutation has previously been shown to render $\mathrm{Cx} 43$ gap junction communication deficient [29]. To confirm expression, we first assessed these cell lines by immunofluorescence, which revealed a dramatic difference in localization when evaluating $\mathrm{Cx} 43$ in the drug sensitive (SK-BR-3) compared to the drug resistant (JIMT-1) cell line. In the drug sensitive SK-BR-3 cells, Cx43 appeared to form large plaques that localized at the plasma membrane between two neighboring cells, indicative of gap junctions (Figure 2A). Conversely, Cx43 in drug resistant JIMT-1 cells was always localized in punctate intracellular structures (Figure 2C). The Cx43 G60S mutant localized in punctate intracellular structures in both the SK-BR-3 and the JIMT-1 cells suggesting loss of GJIC as expected (Figure 2A and 2C). EGFR was used in these experiments to demarcate cells. Though not a measure of activity, this observation suggests that SK-BR-3 cells maintain the capacity to drive Cx43 gap junction plaque formation whereas JIMT-1 cells appear to have lost this capacity, despite both cell lines exhibiting low endogenous expression of Cx43 (Figure 2A and 2C, top panels, Figure 1C, and Supplementary Figure 1).

To directly test the ability of each of the $\mathrm{Cx} 43$ expressing cell lines to propagate GJIC, we performed cell coupling analysis. Our results indicate that overexpression of Cx43 induced coupling in SK-BR-3 cells, indicating that the mechanisms allowing $\mathrm{Cx} 43$ to propagate GJIC is intact in these cells (Figure 2B). Expression of $\mathrm{Cx} 43$ G60S in SK-BR-3 cells did not induce GJIC (Figure 2B). However, when we evaluated the same properties in JIMT-1 cells, Cx43 overexpression did not induce coupling, nor did the Cx43 G60S mutant, suggesting that Cx43 GJIC is impaired in JIMT-1 cells (Figure 2D).
A

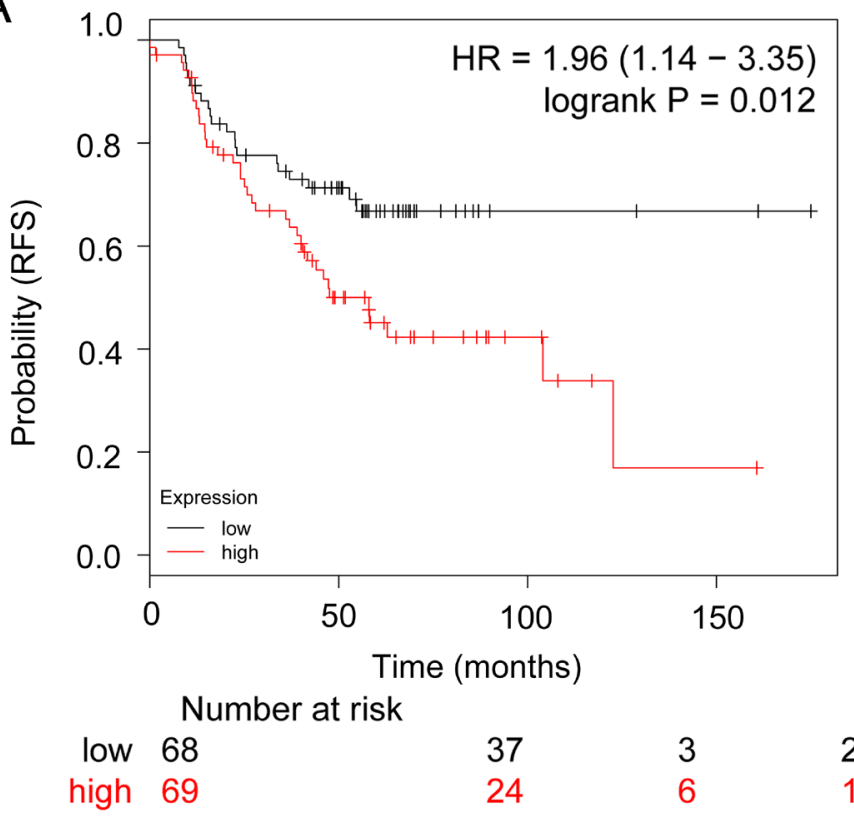

B
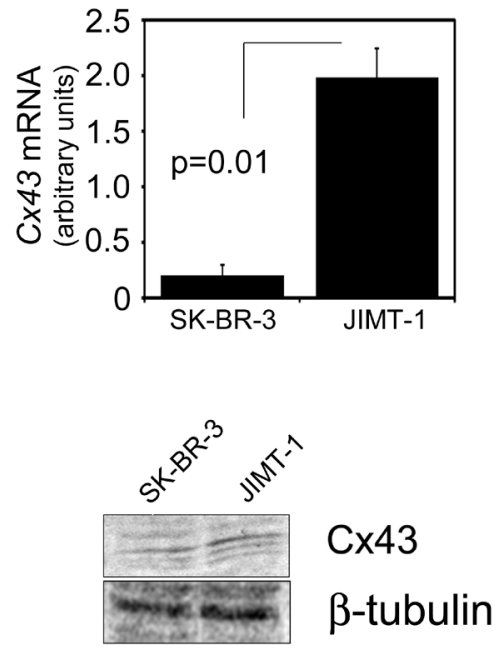

Figure 1: Cx43 (GJAI1) mRNA is elevated in JIMT-1 cells compared to SK-BR-3 cells but Cx43 protein is not. (A) GJA1 expression is associated with reduced relapse free survival (RFS) in HER2 ${ }^{+} /$ErbB2 patients. Gene probe GJA1 201667_at was used for analysis with HER2 ${ }^{+}$status set to "positive" and ER status set to "negative" yielding n=137 patient samples with available clinical data containing the selected events. A total of $\mathrm{n}=68$ patients were scored as "low" GJA1 and n=69 were scored as "high" GJA1. Analysis tool automatically removed redundant samples and excluded any biased arrays. The probe expression range was classified as 73-16584 with a cutoff value of 2320 used for analysis. $H R=1.96$, logrank p-value $=0.012$. (B) Quantitative RealTime PCR assessment of Cx43 (GJA1) mRNA expression levels in SK-BR-3 compared with JIMT-1 breast cancer cells. JIMT-1 cells had $\sim 8$-fold higher levels of GJA1 mRNA in relation with SK-BR-3. GJA1 levels were normalized to GAPDH. Student's T-test indicated a p-value of $\mathrm{p}=0.01, \mathrm{n}=3$. (C) Western blot analysis of endogenous Cx43 protein levels in SK-BR-3 and JIMT-1 cells. 
Taken together, these findings indicate that although Cx43 GJIC is impaired during breast cancer malignancy in HER2+ cells that remain sensitive to HER2 inhibitor, likely due to downregulation of total Cx43 levels, GJIC is rescuable by $\mathrm{Cx} 43$ overexpression. Interestingly, the gap junction activity of $\mathrm{Cx} 43$ protein appears to be further compromised during the acquisition or establishment of resistance in a manner that prevents exogenous expression

A
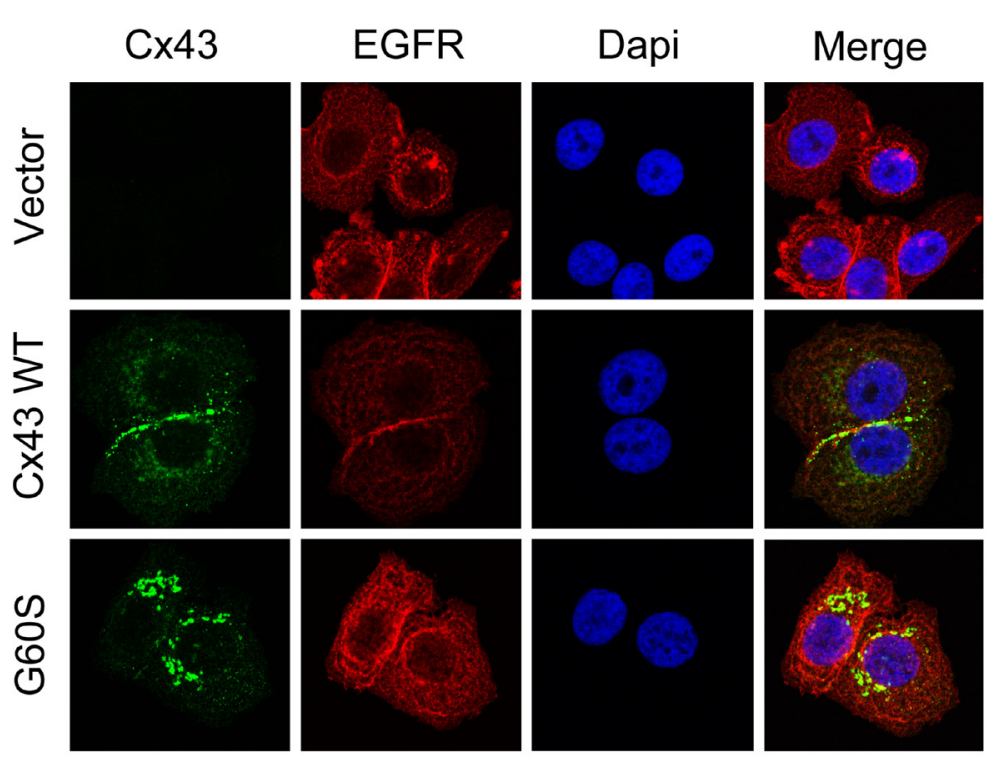

\section{SKBR3}

C

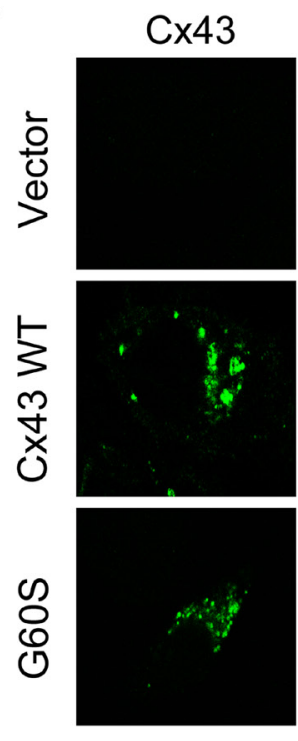

EGFR
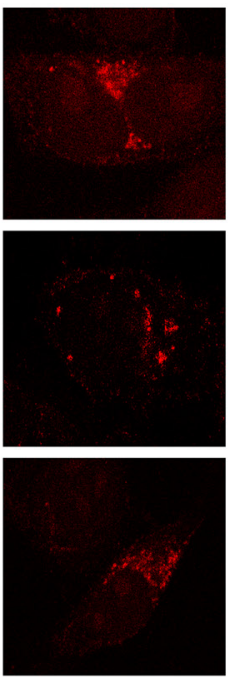
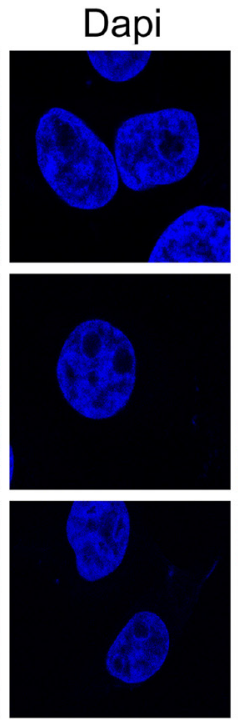

JIMT-1

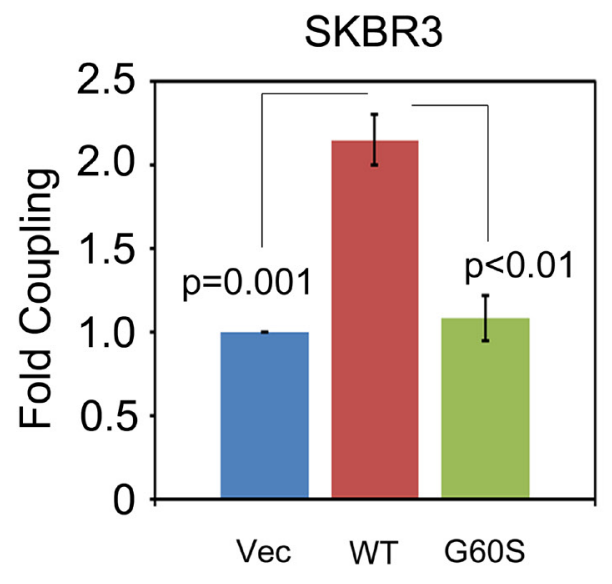

B

D

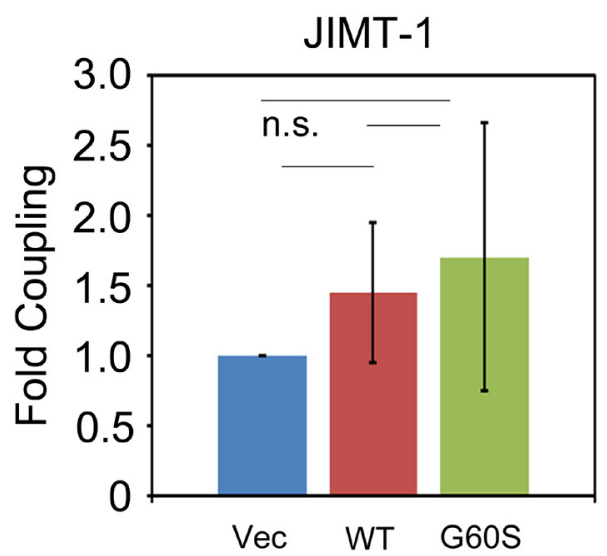

Figure 2: The ability to restore Cx43 GJIC in JIMT-1 cells is compromised. (A) SK-BR-3 cells expressing either a vector control, Cx43, or Cx43 G60S were stained with anti-Cx43 antibody to detect $\mathrm{Cx} 43$ and anti-EGFR antibody to demarcate cells. Nuclei were stained with Hoechst dye. (B) Quantitation of parachute assay comparing SK-BR-3 cells expressing either a vector control, Cx43, or Cx43 G60S. (C) JIMT-1 cells expressing either a vector control, Cx43, or Cx43 G60S were stained with anti-Cx43 antibody to detect Cx43 and anti-EGFR antibody to demarcate cells. Nuclei were stained with Hoechst dye. (D) Quantitation of parachute assay comparing JMT-1 cells expressing either a vector control, Cx43, or Cx43 G60S. 
of $\mathrm{Cx} 43$ from rescuing $\mathrm{Cx} 43 \mathrm{GJIC}$ in HER2 inhibitor resistant cells.

\section{Cx43 expression in HER2 inhibitor resistant cells leads to increased capacity for proliferation}

Since prior reports indicate that $\mathrm{Cx} 43$ regulates cellular functions including proliferation through noncanonical signaling [30-32], we next wanted to determine whether the differences we observed in $\mathrm{Cx} 43$ protein regulation in the HER2 inhibitor sensitive and resistant cell lines affect cellular proliferation and viability. First, we evaluated proliferation by MTT assay in the SK-BR-3 and JIMT-1 control, Cx43, or Cx43 G60S overexpressing cells. We found that $\mathrm{Cx} 43$ overexpression increased proliferation in the JIMT-1 (resistant) cells (Figure 3B) but not the SKBR-3 (sensitive) cells (Figure 3A). The effect of the $\mathrm{Cx} 43$ G60S mutant in each cell line mirrored the phenotype of the wild type (WT) Cx43 (Figure 3A and 3B). Following, we evaluated viability under serum limiting conditions in each cell type. Each cell line was plated in equal number and then subjected to serum deprivation the following day. Total cell number was evaluated prior to serum deprivation ( $0 \mathrm{hr}$ ) and at $48 \mathrm{hr}$ post-serum withdrawal. We found that JIMT-1 cells expressing Cx43 survived and multiplied $\sim 4-$ fold during this period whereas cell proliferation remained static under these conditions in the SK-BR-3-Cx43 cell population (Supplementary Figure 3). We noted that cell number did not decrease in any of the populations under serum limiting conditions, suggesting no inherent loss in viability. Therefore, these findings indicated to us that the dysregulation of $\mathrm{Cx} 43 \mathrm{GJIC}$ activity in the HER2 inhibitor resistant JIMT-1 cells results in increased proliferation.

\section{Non-junctional $\mathrm{Cx} 43$ is a feature of HER2 inhibitor resistant breast cancer cells that drives tumorigenesis}

Based on our findings thus far, we predicted that overexpression of Cx43 in JIMT-1 cells, which remain GJIC inactive, would lead to an increase in breast cancer properties including mammosphere formation and tumor growth. When we tested each of these properties, we found that SK-BR-3 cells had a poor capacity for forming mammospheres, similar to previous reports [33], and that $\mathrm{Cx} 43$ expression did not alter the capacity for mammosphere formation in the SK-BR-3 cell line (Figure 3C). Interestingly, the $\mathrm{Cx} 43 \mathrm{G} 60 \mathrm{~S}$ mutant increased mammosphere formation in the SK-Br-3 cells (Figure 3C). The differential activity of the G60S mutation in the SKBR-3 cells suggested to us that the GJIC-deficient Cx43 could have non-junctional functions that contribute to the mammosphere forming capacity. When we evaluated tumor formation in the SK-BR-3 control, Cx 43, and Cx43 G60S cells using a mammary tumor xenograft assay, the Cx43 expressing SK-BR-3 cells had a reduced capacity for tumor growth compared to vector control cells and $\mathrm{Cx} 43$ G60S cells (Figure 4A). Conversely, Cx43 overexpression in JIMT-1 cells significantly increased primary mammosphere formation ( $\sim 3$-fold increase, $p<0.001$, Figure 3D), as did the Cx43 G60S mutant (Figure 3D). Furthermore, $\mathrm{Cx} 43$ and $\mathrm{Cx} 43$ G60S promoted tumor growth ( 2 -fold increase in volume, Figure $4 \mathrm{~B})$ over vector control expressing JIMT-1 cells. Taken together, these findings indicate that despite $\mathrm{Cx} 43$ expression, dysregulation of $\mathrm{Cx} 43$ promotes tumorigenic properties in JIMT-1 cells, allowing the resulting breast cancers to be more aggressive.

\section{Non-junctional $\mathrm{Cx} 43$ is a feature of HER2 inhibitor resistant breast cancer cells that promotes angiogenesis and migration}

Prior reports suggest a potential role for $\mathrm{Cx} 43$ in regulating breast cancer cell migration and consequently, metastasis [8-15, 19, 34, 35]. Further reports also suggest a possible role for $\mathrm{Cx} 43$ in angiogenesis [36-38]. Therefore, we evaluated these properties in our vector control and Cx43 expressing SK-BR-3 and JIMT-1 breast cancer cell lines. To evaluate angiogenesis, we performed an endothelial tube assay to assess how conditioned medium from vector control and $\mathrm{Cx} 43$ expressing SK-BR-3 or JIMT-1 cells would impact tube formation. We found that the conditioned medium from JIMT-1 Cx3 cell population was able to promote tube formation more robustly than the vector control JIMT-1 cells as well as both the vector and Cx43 expressing SK-BR-3 cells (Supplementary Figure 4).

We next evaluated the migration capacity of each breast cancer cell line by transwell migration assay. Interestingly, the SK-BR-3 cells expressing Cx43 migrated less efficiently than the vector control SK-BR-3, whereas the $\mathrm{Cx} 43$ G60S GJIC-deficient cells did not suppress migration (Figure 4C). However, the JIMT-1 cells expressing $\mathrm{Cx} 43$ migrated more efficiently than the vector control JIMT-1 cells, as did the Cx43 G60S expressing JIMT-1 cells (Figure 4D). These findings suggest that maintaining $\mathrm{Cx} 43$ GJIC inhibits cell migration. Furthermore, as evidenced by results in the JIMT-1 cells, a non-gap junctional $\mathrm{Cx} 43$ can promote metastatic features including angiogenesis and cell migration.

\section{DISCUSSION}

We have investigated differences in $\mathrm{Cx} 43$ regulation in HER2+ breast cancer cells that are drug sensitive and drug resistant to HER2 inhibitors. Based on our observations, we conclude that when experimental methods are used to overexpress $\mathrm{Cx} 43$, whether the breast cancer cell is sensitive to HER2 inhibitors or resistant to HER2 inhibitors dictates if the exogenously expressed $\mathrm{Cx} 43$ can induce the formation of functional 
gap junctions. Based on the findings reported here, we suggest a progressive model whereby HER2+ breast cancer cells can gradually accumulate mechanisms to disable Cx43 GJIC as a feature of drug resistance. We surmise that as the cell becomes transformed, Cx43 expression is reduced, presumably in order to reduce GJIC. However, the cell will likely remain gap junction competent despite the reduced Cx43 protein levels. At early stages of malignancy, it seems likely that cancer cells will retain their ability to communicate through gap junctions, if a signal such as $\mathrm{Cx} 43$ overexpression, is provided to facilitate the formation of gap junctions. However, as the cancer cell develops resistance to chemotherapeutic agents, additional mechanisms arise in order to completely impair GJIC, such as mislocalization of $\mathrm{Cx} 43$. Since $\mathrm{Cx} 43$ protein is directed elsewhere in the cell, non-canonical functions of $\mathrm{Cx} 43$ likely predominate, leading to changes in proliferation and survival that can potentially influence tumor aggressiveness. Further studies to understand the mechanisms that impair gap
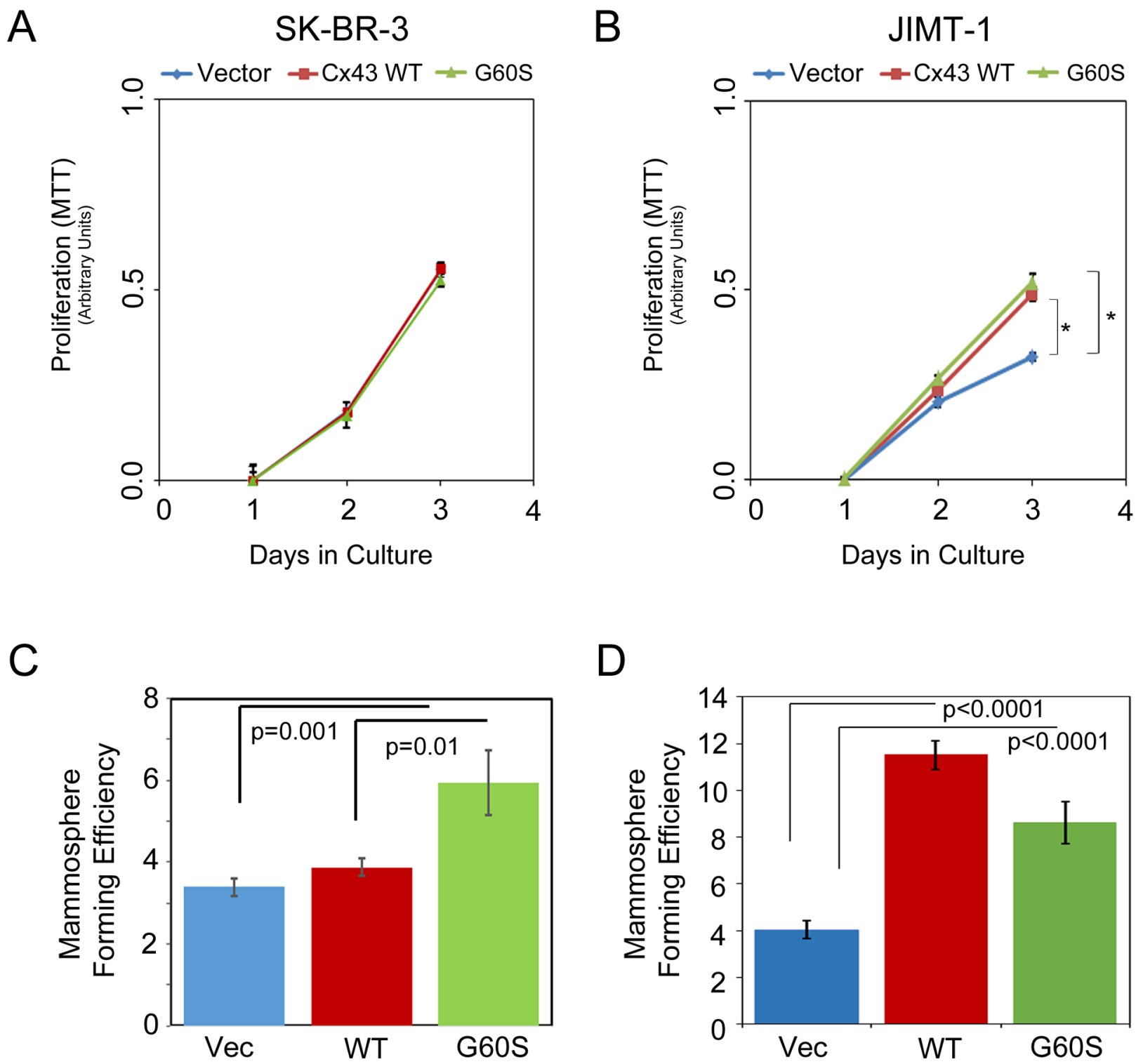

Figure 3: Cell proliferation and mammosphere formation in SK-BR-3 and JIMT-1 cells expressing Cx43. (A) MTT analysis of SK-BR-3 expressing a vector control, Cx43, or Cx43 G60S. Proliferation was assessed over the course of 3 days. (B) MTT analysis of JIMT-1 expressing a vector, Cx43, or Cx43 G60S. Proliferation was assessed over the course of 3 days. (C) SK-BR-3 expressing vector control, $\mathrm{Cx} 43$, or $\mathrm{Cx} 43 \mathrm{G} 60 \mathrm{~S}$ were placed under conditions for assessing mammosphere formation. Mammosphere forming units were quantitated and student's T-test was performed to determine $\mathrm{p}$-values, $\mathrm{p}=0.01$ as indicated, $\mathrm{n}=32$ sample wells per experiment. (D) JIMT-1 expressing vector control, Cx43, or Cx43 G60S were placed under conditions for assessing mammosphere formation. Mammosphere forming units were quantitated and student's T-test was performed to determine p-values, $\mathrm{p}<0.001$ as indicated, $\mathrm{n}=32 \mathrm{sample}$ wells per experiment. 
junction formation in drug resistant breast cancer cells are required in order to determine if it is possible to force the reestablishment of gap junctions in drug resistant cells by methods beyond Cx43 overexpression. Some prior studies have implicated that re-establishment of $\mathrm{Cx} 43 \mathrm{GJIC}$ is required for metastasis [4, 16, 18, 19, 34, 39]. Therefore, great care must be taken to study the activity of $\mathrm{Cx} 43$ in accordance with the different stages of metastasis (i.e. invasion, intravasation, extravasation, and colonization). Likely, extensive studies to evaluate the Cx43 lifecycle

\section{A}

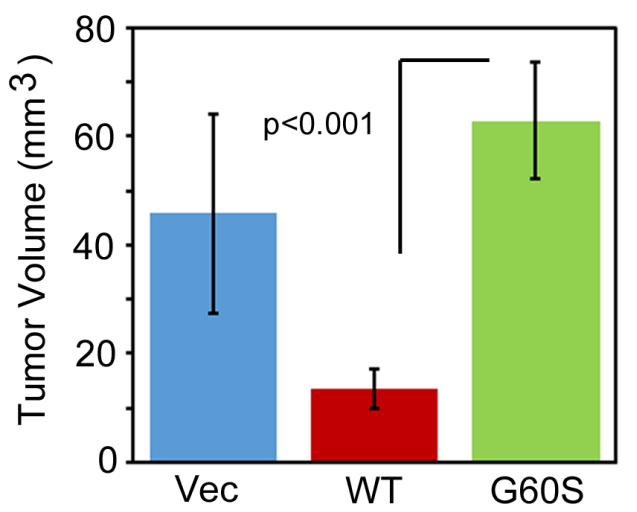

B

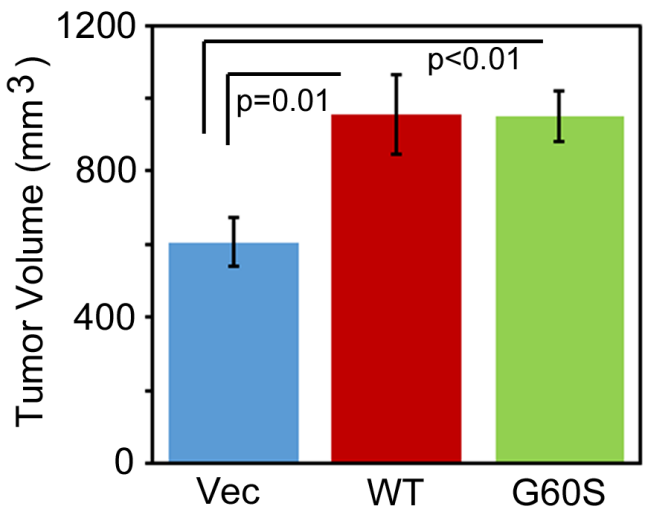

C

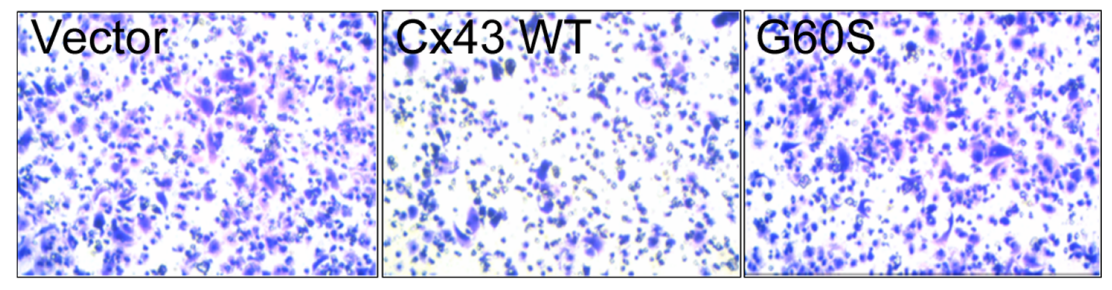

D

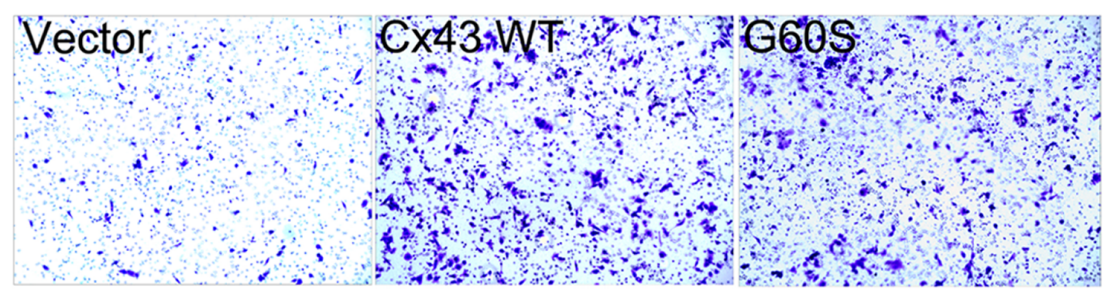

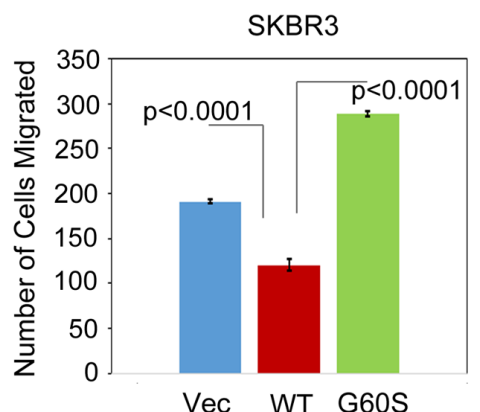

JIMT-1

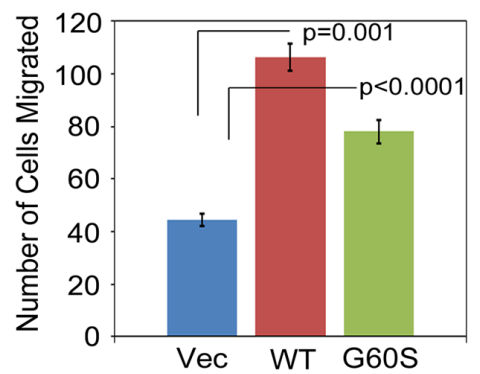

Figure 4: Expression of Cx43 in JIMT-1 cells promotes tumor growth and cell migration. (A) SK-BR-3 expressing vector control, Cx43, or Cx43 G60S were injected into the mammary fat pad of immunocompromised mice to assess mammary tumor xenograft formation and growth. Tumor volume analysis on day 32 post-injection is presented. Student's T-test was performed to determine p-values, $\mathrm{p}<0.001$ as indicated, $\mathrm{n}=10$ animals per group. (B) JIMT-1 expressing vector control or Cx43 were injected into the mammary fat pad of immunocompromised mice to assess mammary tumor xenograft formation and growth. Tumor volume analysis on day 32 post-injection is presented. Student's T-test was performed to determine $\mathrm{p}$-values, $\mathrm{p}=$ or $<0.01$ as indicated, $\mathrm{n}=10$ animals per group. (C) SK-BR-3 cells expressing vector control, $\mathrm{Cx} 43$, or $\mathrm{Cx} 43 \mathrm{G} 60 \mathrm{~S}$ were assessed by transwell assay to evaluate migration. Representative images show that Cx43 expressing cells migrate to a lesser extent than control or Cx43 G60S cells. Number of cells migrated was quantitated and students T-test was used to determine $\mathrm{p}$-values, $\mathrm{p}<0.0001$ as indicated, $\mathrm{n}=4$ samples per experiment. (D) JIMT- 1 cells expressing vector control, $\mathrm{Cx} 43$, or Cx43 G60S were assessed by transwell assay to evaluate migration. Representative images show that Cx43 and Cx43 G60S expressing cells migrate to a greater extent than control cells. Number of cells migrated was quantitated and students T-test was used to determine $\mathrm{p}$-values, $\mathrm{p}<0.0001$ as indicated, $\mathrm{n}=4$ samples per experiment. 
and how this protein is trafficked in models of specific breast cancer subtypes will be required to determine the mechanism by which cells retain Cx43 away from gap junctions.

Importantly, these studies highlight a less well studied area of $\mathrm{Cx} 43$, namely, non-canonical functions for Cx43 that are independent of its gap junctional activity. The C-terminal portion of $\mathrm{Cx} 43$, which is located in the cytoplasm, contributes to many non-canonical $\mathrm{Cx} 43$ functions [20]. This portion of Cx43 represents the main site of interaction with intracellular proteins and has been reported to contribute to cell proliferation (cell cycle), cell death, migration, and transcription [20, 32, 40-42]. The general overall observation from these studies is that the C-terminal domain of Cx43 impairs cell proliferation but promotes cell migration [20]. Our findings support observations that gap junction independent $\mathrm{Cx} 43$ affects these cellular processes. However, we find that $\mathrm{Cx} 43$ expression in JIMT-1 cells increased the proliferative and tumor growth capacity as well as induced migration of these cells. The former is contradictory to the general trend observed in prior studies on the $\mathrm{C}$-terminal domain. Consequently, we must consider whether the C-terminal domain of $\mathrm{Cx} 43$ in JIMT-1 cells is being differentially modified and how this relates to our findings. Albeit we only used a full length protein for our studies but a GJICdeficient G60S mutant of Cx43 confirmed many of our observations. Since it is largely unknown whether the G60S point mutation alters regulation of the C-terminal domain of $\mathrm{Cx} 43$, additional studies are required to evaluate whether regulatory events including proteinprotein interactions are altered. It will be interesting to determine where $\mathrm{Cx} 43$ is acting in the JIMT-1 cells to potentiate these non-canonical functions, what signaling pathways are regulating these functions, and possibly how Cx43 is differentially processed in these cells. Recent efforts have focused on connexin hemichannel function but non-membrane related localization is also reported for Cx43 and thus, we must consider all avenues of function for this protein $[7,20]$.

It is worth noting that distinct cell properties were affected in the SK-BR-3 cells by restoring GJIC through exogenous Cx43 expression. We observed a clear effect on migration in the SK-BR-3 cells. Re-establishing GJIC via $\mathrm{Cx} 43$ expression reduced cell migration and the GJICdeficient G60S mutant of Cx43 did not. This finding is consistent with reports that $\mathrm{Cx} 43 \mathrm{GJIC}$ inhibits cell migration [8-15]. Similarly, an increase in migration was observed in JIMT-1 cells that overexpress Cx43 but lack GJIC. The latter observation does not clarify whether it is the impaired GJIC or non-junctional roles for $\mathrm{Cx} 43$ that promote the cell migration in JIMT-1 cells. These findings further highlight some of the confusion in the $\mathrm{Cx} 43$ breast cancer field, mainly lack of consensus for $\mathrm{Cx} 43$ 's role in metastasis. However, it needs to be noted that metastasis is a complicated process that not only involves the migration of the tumor cell but how the tumor cell interacts with its environment, leaving room for many potential roles that Cx43 and GJIC could play during the metastatic process.

Certainly, we must discuss the merits and faults of using cultured cell lines as well as the positives and negatives of using overexpression as an experimental model system when studying Cx43. While both the SKBR-3 cell line and the JIMT-1 cell line are classified as HER2+, there are distinct differences in their characteristics and culturing methods. The SK-BR-3 line was derived in 1970 by pleural effusion from a 43 year old female with metastatic breast cancer that had been treated with standard chemotherapy whereas the JIMT-1 line was derived by pleural effusion from a 62 year old female with grade 3 invasive breast cancer that had received trastuzumab and developed resistance to trastuzumab [43, 44]. While both cell lines were derived from aggressive HER2+ breast cancers, the treatment protocols each patient received likely contributed to the overall behavior of the resultant cell lines in culture beyond the obvious sensitivity or resistance to HER2 inhibitors. Exogenous expression of proteins is clearly a contrived experimental system. However, overexpression can be used to exacerbate phenotypes driven by the protein of interest, in our case Cx43. Moving forward, identifying reagents to modify endogenous $\mathrm{Cx} 43$ protein may be critical. We and others have performed studies in cancer models using agents such a therapeutic peptides that mimic or antagonize endogenous Cx43 function to study this protein $[7,45,46]$. It is predicted that the ability to evaluate and test protein function for $\mathrm{Cx} 43$ will become more efficient and effective as we increase our knowledge about how this protein is regulated in different cell types and disease states.

\section{MATERIALS AND METHODS}

\section{Cell culture}

All cells were grown at $37^{\circ} \mathrm{C}$ and $5 \% \mathrm{CO}_{2}$. SKBR-3 cells were obtained from American Type Culture Collection $\left(\mathrm{ATCC}^{\circledR}\right.$, Manassas, VA) and cultured in DMEM (Corning 10-017-CV) supplemented with 10\% FBS (Gibco/Thermo-Fisher Scientific), $5 \mu \mathrm{g} / \mathrm{ml}$ Insulin (Gemini Bio-Products, West Sacromento, CA), 2mM Glutamine (Corning), and Penicillin/Streptomycin (Pen/ Strep, Thermo Scientific). JIMT-1 cells were obtained from Addex Bio (Addex Bio C0006005, San Diego, CA) and cultured in DMEM (Corning 10-017-CV) supplemented with 10\% FBS (Gibco/Thermo-Fisher Scientific), 2mM Glutamine (Corning), and Penicillin/ Streptomycin (Pen/Strep, Thermo Scientific). JIMT-1 cells were cultured for less than 6 months since purchase and tested negative for bacteria, mycoplasma, yeast, HIV, Hepatitis B and Hepatitis C (Addex Bio C000605). SVR angiosarcoma cells were obtained from American Type Culture Collection (ATCC ${ }^{\circledR}$, Manassas, VA) and cultured 
in Opti-MEM 8\% fetal bovine serum (FBS) (Omega Scientific, Tarzana, CA). In addition, SVR angiosarcoma cells were tested negative by Research Analytic Diagnostic Laboratory (Columbia, MO) by PCR evaluation for: Ectromelia, EDIM, LCMV, LDEV, MHV, MNV, MPV, MVM, Mycoplasma sp., Polyoma, PVM, REO3, Sendai, TMEV GDVII.

\section{RNA isolation and real time PCR}

RNA was prepared by using the GeneJet RNA isolation kit (Thermo-Fisher Scientific). Reverse transcription was performed using iScript Reverse Transcriptase Supermix (Bio-Rad). The resulting cDNA was used to perform quantitative RealTime PCR using the Bio-Rad myIQ system. PrimePCR SYBR Green Assay for human GJA1 (qHsaCID0012977) was purchased from Bio-Rad. Primers for GAPDH are Forward-TGCACCACCAACTGCTTAGC and ReverseGGCATGGACTGTGGTCATGAG.

\section{Immunoblotting}

Cells were lysed in 2X Laemmli sample buffer followed by sonication (Artek Systems, BioLogics Inc., Manassas, VA) at 30\% amplitude for $10 \mathrm{sec}$. Primary antibodies used for western blotting are: anti-Cx43 (Sigma-Aldrich C6219) and anti- $\beta$-tubulin (Santa Cruz sc55529). Imaging and quantitation was performed on the FluorChem-R instrument (ProteinSimple, San Jose, CA). Quantitation of protein expression was performed using AlphaView software. Cx43 was normalized to $\beta$-tubulin.

\section{Immunofluorescense}

Cells were plated on No. 1.5 square $22 \times 22 \mathrm{~mm}$ coverslips (Corning). Primary antibodies used for immunofluorescence are: anti-Cx43 (Sigma-Aldrich C6219) and anti-EGFR (Santa Cruz sc-373746). Secondary antibodies are Alexa Fluor 488 (ThermoFisher Scientific) and Alexa Fluor 594 (Thermo-Fisher Scientific). Imaging was performed using $63 \mathrm{X}$ oil immersion objective (total magnification 630X) on a Leica TCS SPE confocal microscope and processed using the LAS X software platform (Leica Microsystems Inc., Buffalo Grove, IL).

\section{Coupling assays}

20,000 cells per well were plated into 96 well plates. A separate dish of Cx43 expressing cells, for each representative cell type, either SK-BR-3 or JIMT-1, was loaded with $1 \mathrm{ng} / \mu \mathrm{l}$ calcein-AM (BD Biosciences, Bedford, MA) for $30 \mathrm{~min}$. The calcein-AM loaded cells were washed, trypsinized, and counted. 5000 dye-loaded cells/well were dropped onto the cells plated in the 96 well dish. 6 hrs later, cells were counted and analyzed for calcein-AM fluorescence using a Luna-FL (Logos Biosystems, Annandale, VA) cell counter. For each cell type $n=6$ replicates were evaluated per experiment and each experiment was performed 3 times. Fold change represents the number of calcein-AM positive cells above the original 5000 dye-loaded cells dropped per well.

\section{Proliferation and cell counting assays}

5,000 cells per well were plated into 96 well plates. At the indicated time points, cells were treated with MTT reagent and absorbance read at $570 \mathrm{nM}$ using a Filtermax F5 plate reader (Molecular Devices, Sunnyvale, CA). For cell counting assays, 100,000 cells per well were plated into 24 well plates. The following day, cells were either counted (time $=0 \mathrm{hrs}$ ) or serum deprived by washing and replacing medium with serum free medium. $48 \mathrm{hrs}$ later, cells in serum free medium were counted and cell numbers were analyzed for fold change compared to time $=0 \mathrm{hrs}$ samples. Cell counting was performed using a Luna-FL (Logos Biosystems, Annandale, VA) cell counter. For all assays, MTT and cell counting, $n=6$ replicates were evaluated for each cell type per experiment and each experiment was performed 3 times.

\section{Mammosphere assay}

Mammosphere assay was performed as previously reported [39]. Briefly, 500 cells per well were plated into cell repellent 96 well plates (Greiner Bio-One, Monroe, NC). On day 10 of the experiment, mammosphere structures were quantitated. For each cell type, $n=32$ replicates were evaluated per experiment and each experiment was performed 3 times.

\section{Angiosarcoma tube formation assay}

ECMatrix (Millipore Corp, Billerica, MA) was thawed, diluted, and solidified into wells of a 96-well plate according to the manufacturer's instructions. SVR angiosarcoma cells were serum starved $(2.5 \%$ FBS $)$ overnight and seeded onto the matrix at a concentration of $35,000-40,000$ cells per well $(n=9)$ in $150 \mu \mathrm{L}$ fresh DMEM with $10 \%$ FBS from four different conditions: SK-BR-3 -vector control, SK-BR-3-Cx43, JIMT-1-vector control, JIMT-1-Cx43. Once plated, the cells were incubated at $37^{\circ} \mathrm{C}, 5 \% \mathrm{CO} 2$ for 4 hours. Images were acquired using 4.0x objective lens of EVOS FLc microscope (Life Technologies, Carlsbad, CA). Results were quantified by counting the number of branch points using Image $\mathrm{J}$ Angiogenesis Analyzer software (National Institutes of Health, Bethesda, MD, USA).

\section{Migration assays}

Transwell migration assays were performed as previously described [47]. Briefly, 50,000 cells were 
plated into upper chamber portion of transwell dish in serum free medium. Complete medium containing 10\% FBS was placed in the bottom chamber portion of the transwell dish. Cells were incubated for $24 \mathrm{hrs}$ before membranes were stained, imaged, and quantitated. Images were captured on a Labomed light microscope using a 20X objective (total magnification 200X).

\section{Animal care and xenograft tumor experiments}

Animal care and all animal experiments were performed with the approval and in accordance with the guidelines of the Medical University of South Carolina IACUC. All mice were housed and cared for in the Animal Research Center at Medical University of South Carolina, which is AAALAC accredited facility. Mice were housed in a BSL2 rooming facility for immunocompromised animals. Animals were euthanized by anesthesia overdose with isofluorane in accordance with the Guide for the Care and Use of Laboratory Animals. Protocols were in place for early and humane endpoints in the event that an experimental animal displayed signs of illness, such as poor body condition, lethargy, piloerection, and lack of grooming behavior, prior to the experimental endpoint. To determine when/if animals should be euthanized, tumor measurements and health monitoring of experimental animals was performed regularly by lab and veterinary staff. For orthotopic tumor analysis, $5 \times 10^{6}$ cells were injected in the abdominal mammary fat pat of immunocompromised mice $\left(\mathrm{Nu} / \mathrm{J}-\mathrm{Foxn} 1^{\mathrm{nu} / \mathrm{nu}}\right.$ or NSG, Jackson Labs). Tumors were evaluated by manual palpation using calipers.

\section{Statistics}

For all statistical analyses, $\mathrm{p}$-values for in vitro experiments were analyzed using Student's T-test. $\mathrm{p}<0.05$ was considered statistically significant. As indicated previously in the methods, all experiments were performed in triplicate with multiple replicates (typically $n>4$ ) for each experiment.

\section{Author contributions}

E.S.Y. designed, performed, and analyzed experiments as well as wrote and edited the manuscript. C.B.W., C.J.W, I.V.B., and S.L.P. performed and analyzed experiments. N.K-D. designed experiments as well as assisted in writing and editing the manuscript. All authors have reviewed and agreed to the information presented in this manuscript.

\section{ACKNOWLEDGMENTS}

The authors would like to acknowledge Jennifer Jaroscak, M.D. for her support of S.L.P during her fellowship at MUSC.

\section{CONFLICTS OF INTEREST}

The authors do not report any conflicts of interest.

\section{FUNDING}

This work was supported, in part, by a grant awarded to E.S.Y. from the METAvivor Research and Support Inc. Research in the Yeh Lab is also supported by R01-CA187305 from the NCI (to E.S.Y), as well as previous pilot funds from an American Cancer Society Institutional Research Grant (IRG-97-219-14) awarded to the Hollings Cancer Center at MUSC and from a Department of Defense grant (W81XWH-11-2-0229) at MUSC. This publication was also supported, in part, by the South Carolina Clinical and Translational Research Insitute, with an academic home at the Medical University of South Carolina, through NIH Grant Numbers TL1 TR001451 and ULT TR001450, in the form of a training award to C.B.W.

\section{REFERENCES}

1. Loewenstein WR, Kanno Y. Intercellular communication and the control of tissue growth: lack of communication between cancer cells. Nature. 1966; 209:1248-1249.

2. Kanczuga-Koda L, Sulkowska M, Koda M, Reszec J, Famulski W, Baltaziak M, Sulkowski S. Expression of connexin 43 in breast cancer in comparison with mammary dysplasia and the normal mammary gland. Folia Morphol (Warsz). 2003; 62:439-442.

3. Kanczuga-Koda L, Sulkowska M, Koda M, Rutkowski $\mathrm{R}$, Sulkowski S. Increased expression of gap junction protein--connexin 32 in lymph node metastases of human ductal breast cancer. Folia Histochem Cytobiol. 2007; 45:S175-180.

4. Kanczuga-Koda L, Sulkowski S, Lenczewski A, Koda M, Wincewicz A, Baltaziak M, Sulkowska M. Increased expression of connexins 26 and 43 in lymph node metastases of breast cancer. J Clin Pathol. 2006; 59:429-433.

5. Kanczuga-Koda L, Sulkowski S, Tomaszewski J, Koda M, Sulkowska M, Przystupa W, Golaszewska J, Baltaziak M. Connexins 26 and 43 correlate with Bak, but not with Bcl-2 protein in breast cancer. Oncol Rep. 2005; 14:325-329.

6. Naus CC, Laird DW. Implications and challenges of connexin connections to cancer. Nat Rev Cancer. 2010; 10:435-441.

7. Aasen T, Mesnil M, Naus CC, Lampe PD, Laird DW. Gap junctions and cancer: communicating for 50 years. Nat Rev Cancer. 2016; 16:775-788.

8. Carystinos GD, Bier A, Batist G. The role of connexinmediated cell-cell communication in breast cancer 
metastasis. J Mammary Gland Biol Neoplasia. 2001; 6:431-440.

9. Kandouz M, Batist G. Gap junctions and connexins as therapeutic targets in cancer. Expert Opin Ther Targets. 2010; 14:681-692.

10. Li Z, Zhou Z, Donahue HJ. Alterations in $\mathrm{Cx} 43$ and OB-cadherin affect breast cancer cell metastatic potential. Clin Exp Metastasis. 2008; 25:265-272.

11. Li Z, Zhou Z, Welch DR, Donahue HJ. Expressing connexin 43 in breast cancer cells reduces their metastasis to lungs. Clin Exp Metastasis. 2008; 25:893-901.

12. McLachlan E, Shao Q, Laird DW. Connexins and gap junctions in mammary gland development and breast cancer progression. J Membr Biol. 2007; 218:107-121.

13. Plante I, Stewart MK, Barr K, Allan AL, Laird DW. Cx43 suppresses mammary tumor metastasis to the lung in a Cx43 mutant mouse model of human disease. Oncogene. 2011; 30:1681-1692.

14. Saunders MM, Seraj MJ, Li Z, Zhou Z, Winter CR, Welch DR, Donahue HJ. Breast cancer metastatic potential correlates with a breakdown in homospecific and heterospecific gap junctional intercellular communication. Cancer Res. 2001; 61:1765-1767.

15. Shishido SN, Delahaye A, Beck A, Nguyen TA. The anticancer effect of PQ1 in the MMTV-PyVT mouse model. Int J Cancer. 2014; 134:1474-1483.

16. Cai J, Jiang WG, Mansel RE. Gap junctional communication and the tyrosine phosphorylation of connexin 43 in interaction between breast cancer and endothelial cells. Int J Mol Med. 1998; 1:273-278.

17. Gould VE, Mosquera JM, Leykauf K, Gattuso P, Durst $\mathrm{M}$, Alonso A. The phosphorylated form of connexin43 is up-regulated in breast hyperplasias and carcinomas and in their neoformed capillaries. Hum Pathol. 2005; 36:536-545.

18. Stoletov K, Strnadel J, Zardouzian E, Momiyama M, Park FD, Kelber JA, Pizzo DP, Hoffman R, VandenBerg $\mathrm{SR}$, Klemke RL. Role of connexins in metastatic breast cancer and melanoma brain colonization. J Cell Sci. 2013; 126:904-913.

19. Pollmann MA, Shao Q, Laird DW, Sandig M. Connexin 43 mediated gap junctional communication enhances breast tumor cell diapedesis in culture. Breast Cancer Res. 2005; 7:R522-534.

20. Leithe E, Mesnil M, Aasen T. The connexin 43 C-terminus: a tail of many tales. Biochim Biophys Acta. 2017.

21. Teleki I, Szasz AM, Maros ME, Gyorffy B, Kulka J, Meggyeshazi N, Kiszner G, Balla P, Samu A, Krenacs T. Correlations of differentially expressed gap junction connexins $\mathrm{Cx} 26, \mathrm{Cx} 30, \mathrm{Cx} 32, \mathrm{Cx} 43$ and $\mathrm{Cx} 46$ with breast cancer progression and prognosis. PLoS One. 2014; 9:e112541.

22. Teleki I, Krenacs T, Szasz MA, Kulka J, Wichmann B, Leo C, Papassotiropoulos B, Riemenschnitter C, Moch H, Varga $\mathrm{Z}$. The potential prognostic value of connexin 26 and
46 expression in neoadjuvant-treated breast cancer. BMC Cancer. 2013; 13:50.

23. Grek CL, Rhett JM, Bruce JS, Abt MA, Ghatnekar GS, Yeh ES. Targeting connexin 43 with alpha-connexin carboxyl-terminal (ACT1) peptide enhances the activity of the targeted inhibitors, tamoxifen and lapatinib, in breast cancer: clinical implication for ACT1. BMC Cancer. 2015; 15:296.

24. Jiang G, Dong S, Yu M, Han X, Zheng C, Zhu X, Tong $\mathrm{X}$. Influence of gap junction intercellular communication composed of connexin 43 on the antineoplastic effect of adriamycin in breast cancer cells. Oncol Lett. 2017; 13:857-866.

25. Szasz AM, Lánczky A, Nagy A, Forster S, Hark K, Green JE, Boussioutas A, Busuttil R, Szabo A, Gyorffy B. Cross-validation of survival associated biomarkers in gastric cancer using transcriptomic data of 1,065 patients. Oncotarget. 2016; 7:49322-49333. https://doi.org/10.18632/ oncotarget.10337.

26. Qin H, Shao Q, Belliveau DJ, Laird DW. Aggregated DsRed-tagged Cx43 and over-expressed $\mathrm{Cx} 43$ are targeted to lysosomes in human breast cancer cells. Cell Commun Adhes. 2001; 8:433-439.

27. Talhouk RS, Fares MB, Rahme GJ, Hariri HH, Rayess T, Dbouk HA, Bazzoun D, Al-Labban D, El-Sabban ME. Context dependent reversion of tumor phenotype by connexin-43 expression in MDA-MB231 cells and MCF-7 cells: role of beta-catenin/connexin43 association. Exp Cell Res. 2013; 319:3065-3080.

28. Tomasetto C, Neveu MJ, Daley J, Horan PK, Sager R. Specificity of gap junction communication among human mammary cells and connexin transfectants in culture. J Cell Biol. 1993; 122:157-167.

29. Flenniken AM, Osborne LR, Anderson N, Ciliberti N, Fleming C, Gittens JE, Gong XQ, Kelsey LB, Lounsbury C, Moreno L, Nieman BJ, Peterson K, Qu D, et al. A Gja1 missense mutation in a mouse model of oculodentodigital dysplasia. Development. 2005; 132:4375-4386.

30. Qin H, Shao Q, Curtis H, Galipeau J, Belliveau DJ, Wang T, Alaoui-Jamali MA, Laird DW. Retroviral delivery of connexin genes to human breast tumor cells inhibits in vivo tumor growth by a mechanism that is independent of significant gap junctional intercellular communication. J Biol Chem. 2002; 277:29132-29138.

31. Huang RP, Fan Y, Hossain MZ, Peng A, Zeng ZL, Boynton AL. Reversion of the neoplastic phenotype of human glioblastoma cells by connexin 43 (cx43). Cancer Res. 1998; 58:5089-5096.

32. Moorby C, Patel M. Dual functions for connexins: $\mathrm{Cx} 43$ regulates growth independently of gap junction formation. Exp Cell Res. 2001; 271:238-248.

33. Manuel Iglesias J, Beloqui I, Garcia-Garcia F, Leis O, Vazquez-Martin A, Eguiara A, Cufi S, Pavon A, Menendez JA, Dopazo J, Martin AG. Mammosphere formation in 
breast carcinoma cell lines depends upon expression of E-cadherin. PLoS One. 2013; 8:e77281.

34. Elzarrad MK, Haroon A, Willecke K, Dobrowolski R, Gillespie MN, Al-Mehdi AB. Connexin-43 upregulation in micrometastases and tumor vasculature and its role in tumor cell attachment to pulmonary endothelium. BMC Med. 2008; 6:20.

35. Kapoor P, Saunders MM, Li Z, Zhou Z, Sheaffer N, Kunze EL, Samant RS, Welch DR, Donahue HJ. Breast cancer metastatic potential: correlation with increased heterotypic gap junctional intercellular communication between breast cancer cells and osteoblastic cells. Int J Cancer. 2004; 111:693-697.

36. McLachlan E, Shao Q, Wang HL, Langlois S, Laird DW. Connexins act as tumor suppressors in three-dimensional mammary cell organoids by regulating differentiation and angiogenesis. Cancer Res. 2006; 66:9886-9894.

37. Choudhary M, Naczki C, Chen W, Barlow KD, Case LD, Metheny-Barlow LJ. Tumor-induced loss of mural Connexin 43 gap junction activity promotes endothelial proliferation. BMC Cancer. 2015; 15:427.

38. Zibara K, Awada Z, Dib L, El-Saghir J, Al-Ghadban S, Ibrik A, El-Zein N, El-Sabban M. Anti-angiogenesis therapy and gap junction inhibition reduce MDA-MB-231 breast cancer cell invasion and metastasis in vitro and in vivo. Sci Rep. 2015; 5:12598.

39. Bos PD, Zhang XH, Nadal C, Shu W, Gomis RR, Nguyen DX, Minn AJ, van de Vijver MJ, Gerald WL, Foekens
JA, Massagué J. Genes that mediate breast cancer metastasis to the brain. Nature. 2009; 459:1005-1009.

40. Zhang YW, Nakayama K, Nakayama K, Morita I. A novel route for connexin 43 to inhibit cell proliferation: negative regulation of S-phase kinase-associated protein (Skp 2). Cancer Res. 2003; 63:1623-1630.

41. Behrens J, Kameritsch P, Wallner S, Pohl U, Pogoda K. The carboxyl tail of $\mathrm{Cx} 43$ augments $\mathrm{p} 38$ mediated cell migration in a gap junction-independent manner. Eur J Cell Biol. 2010; 89:828-838.

42. Hebert C, Stains JP. An intact connexin43 is required to enhance signaling and gene expression in osteoblast-like cells. J Cell Biochem. 2013; 114:2542-2550.

43. Trempe GL. Human breast cancer in culture. Recent Results Cancer Res. 1976; 33-41.

44. Tanner M, Kapanen AI, Junttila T, Raheem O, Grenman S, Elo J, Elenius K, Isola J. Characterization of a novel cell line established from a patient with Herceptin-resistant breast cancer. Mol Cancer Ther. 2004; 3:1585-1592.

45. Grek CL, Rhett JM, Ghatnekar GS. Cardiac to cancer: connecting connexins to clinical opportunity. FEBS Lett. 2014; 588:1349-1364.

46. Grek CL, Rhett JM, Bruce JS, Ghatnekar GS, Yeh ES. Connexin 43, breast cancer tumor suppressor: missed connections? Cancer Lett. 2016; 374:117-126.

47. The Transwell Migration Assay. In: Essentials of Cell Biology. JoVE Science Education Database. 2017. 